\title{
A Qualitative Study of Early Intervention Psychosis (EIP) Service Patient's Experience of Sleep, Exercise, Sleep Hygiene Advice and Fitbit Wearable Activity and Sleep Tracker
}

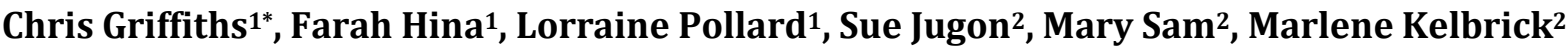 \\ ${ }^{1}$ Innovation and Research department, Northamptonshire Healthcare NHS Foundation Trust, Northampton, UK \\ ${ }^{2} \mathrm{~N}$-STEP Early intervention service, Northamptonshire Healthcare NHS Foundation Trust, Northampton, UK \\ Email: *Chris.Griffiths@nhft.nhs.uk
}

How to cite this paper: Griffiths, C., Hina, F., Pollard, L., Jugon, S., Sam, M. and Kelbrick, M. (2021) A Qualitative Study of Early Intervention Psychosis (EIP) Service Patient's Experience of Sleep, Exercise, Sleep Hygiene Advice and Fitbit Wearable Activity and Sleep Tracker. Open Journal of Psychiatry, 11, 91-106.

https://doi.org/10.4236/ojpsych.2021.112009

Received: March 23, 2021

Accepted: April 19, 2021

Published: April 22, 2021

Copyright $\odot 2021$ by author(s) and Scientific Research Publishing Inc. This work is licensed under the Creative Commons Attribution International License (CC BY 4.0).

http://creativecommons.org/licenses/by/4.0/ (c) (i) Open Access

\begin{abstract}
Background: In psychosis physical activity, sleep, mental health, physical health, wellbeing, quality of life, cognition and functioning are interconnected. People who experience psychosis are more likely than the general population to have low levels of physical activity, high levels of sedentary behaviour and sleep problems. This project was innovative in seeking to address these issues through provision of a wearable activity and sleep tracker (a Fitbit) and sleep hygiene advice. Participants: Participants using an early intervention psychosis (EIP) service took part in an eight-week intervention, which incorporated the provision of a Fitbit, sleep hygiene advice as well as three engagement, feedback and discussion points with a clinician. Methods: A qualitative approach was used to conduct in-depth semi-structured interviews with 12 of the 25 intervention participants ( 5 male; 7 female). Thematic and content analyses were employed to analyse the data. Results: Participants provided valuable insights into their experience of sleep, exercise, Fitbit use and sleep hygiene advice use. It was found that participants placed a high value on effective night time sleep, recognized improvements in physical activity and noted a positive effect on mood and wellbeing as a result of Fitbit use. The negative impact of having ineffective night time sleep and insufficient physical activity was described. Participants demonstrated a good level of understanding of the connection between sleep, exercise, wellbeing, and health. Conclusion: Participants reported the Fitbit and sleep hygiene advice received through an EIP service to be beneficial for improved levels of physical activity and exercise, and more effective sleep. This is a simple and low cost intervention which could be made widely available through EIP and other mental health services.
\end{abstract}




\section{Keywords}

Fitbit, Tracker, Psychosis, Exercise, Sleep, Wearables, Activity

\section{Introduction}

People who experience psychosis have lower levels of physical activity and higher levels of sedentary behaviour than the general population [1] [2]. This is linked to symptoms of depression, lower wellbeing, hopelessness, lower quality of life and physical health conditions (cardio vascular disease [CVD]), stroke, hypertension, osteoarthritis, diabetes, and chronic obstructive pulmonary disease (COPD) [3] [4]. Regular physical activity can improve wellbeing, and is a preventive factor against at least 25 chronic medical conditions [3]. Engaging in physical exercise is associated with improved quality of life, psychotic symptomatology, cognition, functioning and physical health for people with psychosis experience [5]. Mental healthcare providers should offer people with psychosis (especially those taking antipsychotics) a combined healthy eating and physical activity programme [6].

Sleep duration outside of a recommended 7 to 9 hours increases risk of mortality, diabetes, cardiovascular disease, stroke, coronary heart disease, and obesity [7] [8]. Sleep disorders are very common in people who experience psychosis (rates of $80 \%$ reported) [9] [10]. Sleep hygiene advice for sleep problems in psychosis may be beneficial [9] [10].

Low levels of physical activity and poor sleep in psychosis may be a linked [9]. Ineffective sleep is negatively linked to psychotic symptom severity, neurocognitive deficits, peripheral immune system measure alterations, memory consolidation, quality of life, rates of help-seeking, and suicidality [9] [10]. DSM-5 recommends that sleep problems should be assessed, monitored and treated when present with psychiatric comorbidities [11].

Using a wearable tracker such as a Fitbit is helpful to increase physical activity, self-awareness, motivation and goal-setting [12]. Fitbit sleep detection is $95 \%$ accurate [13]. People with serious mental illness have found Fitbit to be acceptable, motivating, and useful for enabling goal setting and healthier lifestyles [14]. Good feasibility and acceptability of using consumer sleep monitoring technology (e.g. Fitbit) in psychosis has been reported [15].

The interviews in this study were conducted with patients in an early intervention psychosis (EIP) service who took part in an eight week intervention incorporating the provision of a Fitbit, sleep hygiene advice and three contact points involving engagement, feedback and discussion with a clinician. The aim of this study was to understand participants' experience of the intervention, and their experience of sleep and physical exercise in the context of their lives and early or first episode psychosis. 


\section{Methods}

\section{Participants}

The sample was recruited purposefully from people using an EIP service within a United Kingdom (UK) National Health Service (NHS) Trust. Participants were included if they were aged 18 or over, had the mental capacity to consent, provided informed consent and had the ability to understand verbal English. EIP service staff reviewed patient notes to check eligibility prior to sending participant information sheet (PIS). Approval for the study was gained from the NHS Trust on 25 August 2020.

12 participants (5 males; 7 females) with an age range of 19 - 54 years old ( $M$ $=30.25 ; \mathrm{SD}=9.92)$ took part in the interview process. Participants identified themselves as White British $(n=6)$, Indian or British Indian $(n=2)$, British or mixed British $(\mathrm{n}=1)$, unknown $(\mathrm{n}=2)$, other $(\mathrm{n}=1)$. All participants were diagnosed with first or early psychosis. Diagnoses included: acute and transient psychotic disorder $(n=3)$, unspecified nonorganic psychosis $(n=5)$, mania with psychotic symptoms $(\mathrm{n}=2)$, bipolar affective disorder $(\mathrm{n}=1)$, delusional dis$\operatorname{order}(\mathrm{n}=1)$.

Setting

The intervention was implemented whilst participants were under the care of the EIP service. In this service every person is offered a National Institute for Health \& Care Excellence (NICE) concordant package of care as per national standards [16].

Timing

Project was undertaken during autumn and winter 2020 when societal restrictions due to COVID-19 were in place.

\section{Intervention}

Twenty five EIP patients were offered an eight week intervention which incorporated the provision of a free Fitbit, sleep hygiene advice as well as three patient engagement, feedback and discussion points with a clinician.

Procedure

Semi-structured telephone interviews were undertaken with 12 participants based in the community under the care of the EIP service. Interviews were carried out over the phone, participants taking the calls at home. Subject areas: 1) physical activity and impact on sleep and wellbeing; 2) physical activity barriers and facilitators; 3) Fitbit impact on activity; 4) sleep quality impact on activity and wellbeing; 5) effective night-time sleep barriers and facilitators; 6) Fitbit impact on sleep; 7) links between activity, sleep, health and wellbeing. Informed consent was taken from all interviewees and they were provided with the right to withdraw at any time. Following transcription, all transcripts were anonymised and the audio files were deleted. Interview duration averaged 30 minutes.

The interview schedule included 3 main parts: 1) Fitbit; 2) sleep; and 3) physical exercise, each with sub-questions:

1) Fitbit: About the Fitbit; charging, frequency of use, usage of apps and display information. 
2) Sleep: Sleep patterns pre and post intervention, the impact of Fitbit use on sleep, mental health and wellbeing, current antipsychotic medication and its effects on sleep, if any.

3) Physical activity: exercise patterns pre and post intervention, barriers to exercising, helpful factors in encouraging to do exercise, the impact of Fitbit use on physical exercise, mental health and wellbeing. Any other comments.

\section{Analysis}

Content analysis was conducted on interview questions which had a yes/no response. Results are displayed in the form of percentages (Table 1).

Thematic analysis was carried out using the following the procedure [17]. Firstly, the researchers familiarised themselves with the material by reading and re-reading the transcripts. Secondly, initial codes were generated line-by-line by two researchers working independently and emerging themes were listed on the basis of similar ideas and patterns running throughout the interviews. Finally, these themes were integrated into overall themes and, where appropriate, each of these themes was further categorised into subthemes. Two researchers collaborated to refine, label and finalise the themes presented in Table 2. Quotations representing and supporting the themes were collated and reviewed; quotes that best represented the themes were selected.

\section{Results}

\section{Thematic analysis findings:}

Two major themes were identified. Theme 1: "The positive impact of Fitbit" and Theme 2: "Perspectives on sleep and physical exercise".

Theme 1: The positive impact of Fitbit

This theme split into the following subthemes: 1) sleep; 2) exercise; 3) feedback and motivation; 4) mental health; and 5) usefulness of Fitbit apps.

1) Sleep

Table 1. Content analysis findings.

\begin{tabular}{lc}
\hline \multicolumn{1}{c}{ Question } & Percentage answered yes \\
\hline Did you have any problems wearing the Fitbit? & $0 \%$ \\
Did you wear it all the time? & $100 \%$ \\
Did you manage to keep it charged? & $100 \%$ \\
Did you use the information on the Fitbit display? & $83 \%$ \\
Did you access Fit bit apps? & $50 \%$ \\
Before you started using the Fitbit did you have any problems: & \\
Getting to sleep? & $8 \%$ \\
Waking up during the night time? & $42 \%$ \\
Not getting enough night time sleep? & $17 \%$ \\
Sleeping during the day that was more than a single 15 minute nap? & $17 \%$ \\
Did you use the sleep hygiene information sheets? & $25 \%$ \\
Do you take anti-psychotic medication? & $100 \%$
\end{tabular}


Table 2. Themes and subthemes.

\begin{tabular}{|c|c|c|}
\hline Major & Key themes & Sub themes \\
\hline \multirow{3}{*}{$\begin{array}{l}\text { 1) The positive } \\
\text { impact of Fitbit }\end{array}$} & a) Sleep & $\begin{array}{l}\text { i) Changes to more effective sleep } \\
\text { ii) Fitbit providing an objective measure } \\
\text { of sleep resulting in reassurance }\end{array}$ \\
\hline & b) Exercise & $\begin{array}{l}\text { i) Fitbit provided information, awareness } \\
\text { and feedback } \\
\text { ii) Fitbit provided motivation }\end{array}$ \\
\hline & c) Usefulness of Fitbit apps & \\
\hline \multirow{3}{*}{$\begin{array}{l}\text { 2) Perspectives on } \\
\text { sleep and } \\
\text { physicalexercise }\end{array}$} & a) Sleep & $\begin{array}{l}\text { i) Applying sleep hygiene for effective sleep } \\
\text { ii) Barriers to effective sleep } \\
\text { iii) Impact of sleep on mood and wellbeing }\end{array}$ \\
\hline & b) Exercise & $\begin{array}{l}\text { i) Facilitators and barriers associated } \\
\text { with exercising } \\
\text { ii) Impact of exercise on mood and wellbeing } \\
\text { iii) COVID restrictions impact on exercise }\end{array}$ \\
\hline & $\begin{array}{l}\text { c) Understanding links } \\
\text { between sleep, exercise, } \\
\text { wellbeing, and health }\end{array}$ & \\
\hline
\end{tabular}

The use of a Fitbit had a positive impact on sleep for many participants. Two sub-themes emerged: a) changes to more effective sleep and b) Fitbit providing an objective measure of sleep resulting in reassurance.

a) Changes to more effective sleep

Patients reported changes undertaken led to more effective sleep; for example, application of sleep hygiene, effect of monitoring as well as receipt of sleep feedback using the Fitbit. These improvements are illustrated by participants' extracts below:

"I would say it's been very positive. I ended up reading a book, I think it's 'Why we sleep' by Matthew Walker because I started getting exposure to the sleep principles, then coming off my medication and sleeping much better so, it has been beneficial. I would say it was more consistent" P01.

"Well, I certainly think, by the end of it, it got improved. I think it gives you a poor, fair or good-I started to get more good and fair sleeps. I have had a few poor ones too but that stopped by the end of it. I think, sort of, you put it on and you think ah I better try and go to sleep. Yes, cos you get a result for your sleep and you try to achieve that result" P116.

"Yes, I have got access to Fitbit apps. I used them to monitor my sleep. My sleep is reduced because before I was sleeping like 13 - 14 hours. Now, I manage sleep well. It's helped me understand my sleep better" P107.

"Oh yes my sleep has definitely improved, I sleep more now and feel well rested. Yes, it's a clever device. It's really good' $\mathrm{P} 03$.

"It helps, makes me. My sleep is improved in the last couples of weeks. It's getting better and I think it's because I am worrying about things less about sleep 
and things are starting to happen how they should. Otherwise, you are worrying and worrying makes things worse. Cos I am seeing the results, it's making me think better and sleep better" P10.

"I think it's good to keeping track of my sleep and things like that. Well, keeping track of my sleep has helped me to do another sleep challenge to do with essential oil so watching it on Fitbit has prompted me to try my scores up, my sleep scores" P02.

"It definitely has an impact especially, on sleep" P24.

"I think it has helped me stabilise" P01.

b) Fitbit providing an objective measure of sleep resulting in reassurance

Some patients expressed concern over inadequate night time sleep. For them, the Fitbit provided an opportunity for an objective measurement over quantity and quality of sleep attained. This opportunity provided reassurance to participants who reported better sleep, and more positivity to continue the endeavours of their day.

"It did have an impact with my psychosis and mental health-seeing like how much I was sleeping, reassuring, trying mindfulness and stuff. In the end, it definitely helped, yes" P01.

"I think knowing that you are having enough, sufficient sleep cos sometimes you don't know if you have had enough sleep. It kind of helps, I guess helps knowing... Like ok $\mathrm{Pm}$ having enough sleep. I guess it's seeing the facts and reassures you that ok, I had 2 hours deep sleep" P06.

"It's given me confidence on sleeping enough. And when I had a bad night sleep and I look at the Fitbit and it's actually not that bad. It's just obviously sometimes, you wake up from deep sleep and feel a bit tired and think you haven't been sleeping very well but having look at the Fitbit, it makes you think, ah wow, you have had 7 hours of sleep. It kind of lifts my spirit that I have slept properly even though I thought I hadn't. It's just that I don't focus on not getting enough sleep and so, negative thinking. It makes me a little more positive. It makes a bit happy like ah cool, and I get on with the day" P10.

2) Exercise:

Fitbit use provided both information and motivation to engage in physical exercise. This is captured in the following two themes.

a) Fitbit provided information, awareness and feedback

People found the Fitbit useful as it provided them with valuable exercise information, awareness and feedback:

"Steps wise. It has helped to track how many steps I had" P02.

"Well, it made me conscious how much I am doing a day like steps wise and calories and that. It's just made me more aware of what I am doing" P10.

"It has made me feel more mindful about doing exercise" P08.

"You can log in your food as well. You know that's quite helpful on the same device. You have exercise on it, food, sleep and social aspects too. It's quite helpfup' P06. 
b) Fitbit provided motivation

Fitbit provided motivation to exercise:

"It motivated me to get more exercise, tells me how many steps I have done and keeps me going. And obviously, tells me how many calories I have burnt' P03.

"It's helped me improve my steps during the day by monitoring it" P107.

"Because you see calories counter, distance travelled and all those aspects, it definitely encourages you to move basically and exercise. When I was wearing the Fitbit and even going to the gym at like 6, sometimes 7 am, $I$ was always using it to see the stats. I also had a few bike rides and I used it" P116.

"It definitely had the impact. Once you know the information, you try to achieve by like walking further" P116.

"It motivates me to get more exercise done like I go for a walk twice a day may be so, the Fitbit motivates me to get fit, to get my steps up and how many calories I have burnt. It's really motivating me" P03.

"Well, ever since I got the Fitbit, I do walking more and catch the 5k thing. So, it helps me track my runs and stuff" P12.

"Well, definitely positive. I even put a set goal on the weight section. So, I try to-my goal was to go down to 12 stones and I achieved it - that was thanks to the Fitbit app. You can put in your weight, what you are and then you set your goal to lose stone or whatever it is and you try to achieve it. So, it's very good" P116.

"You know it's like when you have done certain amount of steps, it's kind of celebrates for you on your watch. It, then you know, I feel like let's see if I can reach the next point where I can celebrate like vibrate celebration like things. So, it makes you do more..." P06.

"Yes, it gives me motivation and for me to improve" P11.

"I show it to my mates like showing how many steps I have done compared to them cos they can't even check cos they don't have it" P170.

"It's nice when you get that buzzing feeling when you have achieved something" P24.

"It definitely made me more motivated to try and do more" P01.

c) Usefulness of Fitbit apps

Fitbit apps were a feature that some patients found played a useful role. For example:

"...mindfulness - I did a little bit of that breathing technique, and I used the headspace app, quite handy" P116.

"Oh yes, I have got access to Fitbit apps. I used them to monitor my sleep" P27.

"I think having physical or meditation thing on there... it helps you to wind down or whatever" P06.

"Yes, I have got access to Fitbit apps. I used them to monitor my sleep. My sleep is reduced because before I was sleeping like 13 - 14 hours. Now, I manage sleep well. It's helped me understand my sleep better" P107. 


\section{Theme 2: Perspectives on sleep and physical exercise}

The second major theme identified was participants' perspectives on sleep and physical exercise.

This was further categorised into three themes and further subthemes:

1) Sleep: a) Applying sleep hygiene for effective sleep; b) Barriers to effective sleep; c) Impact of sleep on mood and wellbeing.

2) Exercise: a) Facilitators and barriers associated with exercising; b) Impact of exercise on mood and wellbeing; c) COVID-19 restrictions impact on exercise.

3) Understanding links between sleep, exercise, wellbeing, and health.

1) Sleep

Patients generally reported a good understanding of the value of sleep and sleep cycles, and impact on various aspects of an individual's life.

a) Applying sleep hygiene for effective sleep

There were several sleep hygiene factors that participants reported were helpful to achieve an effective night's sleep. For example, sleep hygiene based routine prior to night time sleep, camomile tea, room temperature conducive to sleep, listening to relaxing music, and engaging in physical exercise to facilitate physical tiredness. These and other factors are illustrated below:

"Trying to have a consistent sleep schedule because that helps to manage the anxiety I get. And then things like reading instead of being on screen" P01.

"Basically, just relax in the evening like chill out. You know watch movie or read a book... relaxing music during the evening helps you to get to sleep. And exercising during the day" P06.

"Having a bath before going to bed" P08.

"I do drink hot chocolate before going to sleep" P12.

"Well, having a bit of an exercise before I go to bed like not straightaway before I go to bed but like gym or football 3 - 4 hours before, it will help me to go to sleep" P170.

"I used to drink camomile tea, yes that actually helps me get to sleep. It has those sleep benefits in it" P170.

"I used to drink camomile tea or similar" P24.

"Well, good temperature. Music helps. Sometimes, I watch something and I sleep or if I listen to music, I sleep" P12

" $A$ lot of it was like turning off screen before sleep, you just try to shut down and like mindfulness - I did a little bit of that breathing technique, and I used the headspace app, quite handy. Basically, it's like shutting down from work, which I am always kind of struggling with" P116.

"I would say it been very positive. I ended up reading a book, I think it's 'Why we sleep' by Matthew Walker because I started getting exposure to the sleep principles" $\mathrm{P} 01$.

"I try and stay away from technology, my mobile phone, for instance. If you watch tele or go on your phone before sleep, it kind of keeps you awake. So, I try and stay away from technology before I go to bed' P03. 
"It was like looking at your screen time and general sleep hygiene. Over time, I have this in my mind that its lots of things but then I did start following them slowly" P01.

"And avoiding playing games or anything too exciting after 9 o clock" P24.

b) Barriers to effective sleep

Patients' also discussed the elements that prevent night time sleep. These included not engaging in physical exercise during the day, excessive screen time whilst using technology shortly before night time sleep, stressors, noise, and intrusive/worrying/negative thoughts. These are illustrated by patients' quotes below:

"Not exercising in a day enough" P01.

"Thoughts like sometimes you are thinking about something or your neighbours, if there is lots of noise" P06.

"I try and stay away from technology, my mobile phone, for instance. If you watch television or go on your phone before sleep, it kind of keeps you awake. So, I try and stay away from technology before I go to bed' P03.

"If I am stressed I guess like exams or anything. Or if it is generally anything is happening in my life, I guess" P12.

“Stress, worrying. Yes, usually work related" P10.

"Ah just general noise, really. Like if something is going on outside in the street or if I am playing too much games, that might affect my sleep" P170.

"The other thing is watching news late night-certainly during the last year. You probably thinking about things, getting annoyed at things that really don't affect you. So, it's quite waste of a time-like even before taking part into the Fitbit study, I used to have sky news, BBC news, all that stuff with notifications in my phone-every 5 minutes, they will make a little noise so I turned it off months ago like end of April/May cos I was getting so stressed of the pandemic. Turning off, switching off your phone as well as social media and all that. I find-I think it's easy for people to fall into that. Stress like, things that really don't affect us in reality" P116.

c) Impact of sleep on mood and wellbeing

Ineffective sleep was reported to have negative impact and, conversely, effective sleep a positive impact on wellbeing.

"Yes, it [sleep] definitely affects mood. You can get stress easily if you haven't had a good night sleep" p116.

"It can affect your mood cos I have been there where you only have 2 hours of sleep and you have to wake up and get to work next week. It can affect your mood so, if you haven't had enough sleep, so, you are a bit moody like you want to go to sleep and if you have had too much sleep, you are a bit tired so, it can affect your mood too. Like people can get on your nerves when you are tired" $\mathrm{P} 170$.

"I have a much better mood If I have a good night sleep, more happy, more energy, I suppose-if I have a good night sleep" P02.

"My mood is definitely lower. Work is harder than it should be. Yes lack of 
sleep is definitely not good" P24.

"I find if I don't have enough sleep, basically, it kind of effects everything else like how you work, what you doing that day or you know has an effect on your mental wellbeing" p06.

“Oh lot! If I don't sleep, I don't get on very well” P08.

"Well, I think sleep is more important than we think it is, I suppose. You don't realise that. Like your brain functions better, you feel obviously more motivated-that's for sure. Certainly with exercise, you sleep better when you exercise" P116.

"I feel like if I don't sleep enough, I feel tired and like lack of energy. I feel a bit down. Whereas, if I get more sleep, I am more active and can focus on things better" P12.

"Definitely a lot. If I don't sleep well, it ruins my day. It makes me more huffy, bit like a hangover really" P10.

"I think sleep is very important. It can get me anxious if I know that I am not going to get good sleep" P24.

2) Exercise:

Reporting from personal experiences, participants demonstrated an excellent understanding of the factors associated with exercise and its impact on mood and wellbeing.

a) Facilitators and barriers associated with exercising

Factors that facilitated exercise could be personal ones (e.g. motivation to stay active and healthy), social factors (e.g. support from friends, family, partner), or environmental (e.g. good weather).

"I have found it beneficial to do live yoga classes than as supposed to the ones on YouTube and tutorials. It's just nice when other people are doing it with you" P01.

"My brother is very fit and athletic I suppose and I live with him. He is quite like you should go for a run so he pushes me but other than that, I suppose due to COVID and stuff, you don't see many people. It's down to you-you being motivated" P116.

"The social aspects of it-that would help. Yes I think those sides of things help. Other people knowing you are trying to achieve fitness ... and that when you are going for a walk' P06

"I have family members who would say if I will come and have a walk with them" P06.

“Well, you just go out when it's not raining" $\mathrm{P} 06$.

"Yes, my partner is quite motivating yes, he helps quite a lot cos he is a sport coach as well. It's just very encouraging really" P02.

"It makes me feel better afterwards, so the motivation, yes" P08.

"Well, my parents tell me to get more exercise so they help. Yes they tell me to go for gym, for instance or go for walks. So they help me a lot when it comes to that. They motivate me" $\mathrm{P} 03$.

"My mum encourages me to exercise. My mates encourage me to play football 
and I encourage myself to keep moving to be honest. That's it' P170.

"My partner; without her, I would definitely be doing less. She is doing something nearly every day and she is trying to get me out sometimes. I am not always motivated to do it but certainly, if she suggest, I would do it for her to make her happy-you know what I mean like we are doing something nice together. And if the weather is good, I would like to go with my friends_skateboarding or bike riding when the spring comes" P24.

Participants reported barriers faced when engaging in physical exercise, these included poor weather, being overweight, tiredness and work. As illustrated below:

"I don't want to do exercise in the evening because I am like tired out of the day or I don't want to wake up in the morning necessarily. Tiredness is the biggest barrier" $\mathrm{P} 01$.

"Energy levels-you don't feel like doing a lot. That's the other thing. I can't really think of. Oh sometimes, also finding time to do it" P06.

"It's myself because I have put too much weight on and I d like to lose it. So Id like to do exercise" P11.

"I think I am progressing well but the barriers are work at the minute. Like by the time I get home, its dark so kind of be put off by it" P08.

"The weather impacts me" P03.

"When it's cold and windy, I find it hard to get out" P24.

"Sometimes, I feel like I m too tired not because I haven't had sleep but because I lack energy. Also, the weather, when it's cloudy and dark, I won't exercise" P12.

b) Impact of exercise on mood and wellbeing

Participants perceived exercise to have a positive impact on mood and wellbeing. As an example, a value was placed on having something physical to focus on.

"I feel better after exercise" P11.

“It makes me feel better, feel like energised" P08.

"I think it gives me more positive energy for the day and I feel energised when I am doing my work and I feel motivated when I am speaking to other people as well. I feel like it makes my mood better too" P12.

"Keeps me fit, healthy, in shape, good fresh air working outside and keeps me focussed on like when I am not in work and in holiday, it's quite negative and almost depressing being stuck at home when everyone else is working and all I do is sitting at home playing $X$-box, got nothing to do. Makes me more relaxed feel like I have achieved something, and I guess there is an element of happiness because you know that the body needs exercise, to work properly" P10.

"I do feel better for it. I think I can sleep better and it helps with my mood as well. It improves my mood. Appetite is a bit better, motivation improves too. I feel good about it like something is right when so many things are strange at the moment" P24.

“Well, it makes me feel better, makes my mood good. A lot better in generaP' 
$\mathrm{P} 03$.

" $A$ h, I think it can affect your mood cos if you are not moving then it can be difficult for you to like don't know get your point across sometimes. Like if you are not moving around enough so, physical fitness that side of things can make you tired and if you are tired and don't get enough sleep then you can be a bit fed up of things. That's that of things but if it s just physical fitness, it can affect your mood cos of endorphins or something. I don't know if its endorphins but some kind of chemical that is released in your brain when you exercise. So, that can affect your mood" P170.

"It has an effect like it puts your mind on rest. You know when you are exercising or you are walking, you are not worrying about anything, you are just doing whatever exercise you are doing. For me, that's a good thing, yes. It's just relaxing sometimes" P06.

"Well I suppose, if I have gone for a walk, I feel more positive, I have got more energy" $\mathrm{P} 02$.

c) The impact of COVID-19 restrictions on exercise

COVID-19 had a negative effect on people's physical movement, social contact, and meaningful activity. The following impact of COVID-19 on exercise was reported by participants:

"I used to go to the gym but I don't go anymore cos of the lockdown so, that's a barrier" $\mathrm{P} 02$.

"During the corona virus, there was obviously gym closed. There wasn't much you can actually do physical exercise wise apart from walk. So I was just literally walking for longer. Yes, that's only thing I could really do" P06.

"I suppose due to COVID and stuff, you don't see many people. It's down to you-you being motivated, look after yourself, sleep well then you stand more chance to exercise well- and generally feel well overall' P116.

"Not exercising in a day enough, which obviously, I think is quite hard because of COVID. Yes, I think that's the main thing" P01.

"Gyms are closed so, I go for walks, twice a day roughly" P03.

"I used to go to the gym when they were open. But ever since gyms are closed, I don't do any physical activity. Walking sometimes" P12.

"It's quite negative and almost depressing being stuck at home when everyone else is working and all I do is sitting at home playing $X$-box, got nothing to do" $\mathrm{P} 10$.

"I think getting back to gym once they had opened would help. I prefer using weights and things; indoors, machines, spacious space and muscles-different groups working out. Swimming as well-I like swimming but that all closed" P24.

3) Understanding links between sleep, exercise, wellbeing, and health

Participants were questioned as to their thoughts about the links between sleep, exercise, wellbeing, and health. As illustrated by the extracts below, participants demonstrated a very good understanding of the interconnected relationships between these factors: 
"Physical exercise is as important as sleep, if not more. It all goes hand in hand. You do need a good night's sleep, you do need exercise. You probably don't need intensive exercises. If you stop exercise like I did for a while, you need a motivation to start it and it does have a direct impact on your mental health, if you are physically well as well. Look after yourself, sleep well then you stand more chance to exercise well-and generally feel well overall' P116.

"If I sleep better, I eat better and then I do exercise better. It would give me more energy and that's how I would describe it. I think they are all kind of interconnecting" P01.

"If you don't do any exercise, don't sleep well, you don't feel well. It also affects your mental health and you don't feel good about yourself. If you don't feel good about yourself, you really don't make any effort on anything. You work is affected as well and your personal life too, you know you got to look after yourself? P116.

"It's really helpful you exercise and like having a balance of all aspects of your wellbeing so, it s like having the right amount of sleep and the right amount of exercise and the right amount of food" P06.

"Well they are all connected to each other because if you exercise, you sleep better and you reduce [increase] endorphins which helps lifting your mood up" P107.

"Yes I feel better when I sleep and exercise well, I feel better in myself and feel confident too. Sometimes, I can't think and speak properly but when I sleep well, I do it properly" $\mathrm{P} 11$.

"Yes if I sleep better at night, it makes me exercise more and that affects my wellbeing, I feel positive" $\mathrm{P} 03$.

"I feel like when the mixture of sleep and exercise is good, my life is more positive and I feel better in general. But I can't do one without the other like I can't do exercise without sleeping and I can't make sleep well without exercising. They both affect wellbeing in a positive way" P12.

"I think they are all very important and they work closely together like, basically like eating and drinking you got to do both to survive and you need exercise to get good sleep and exercise and sleep makes you feel better your wellbeing entirely" P10.

"Yes so, I you don't get enough exercise, you might not be tired enough to go to sleep and then in turn, you might stay up late and after that, you might be a bit tired like towards other people so, it's important to get exercise so that you wake up refreshed and ready for the next day" P170.

"I think I can sleep better and it helps with my mood as well. It improves my mood. Appetite is a bit better, motivation improves too. I feel good about it like something is right when so many things are strange at the moment" P24.

"I believe they both play a really important role with wellbeing. Like when I come, if I have had a healthy food, go for a run, sleep a bit earlier, Ill feel really good the next day whereas, if I had stayed late, had alcohol, Id feel bad' P24. 


\section{Discussion}

Patients using an EIP service provided valuable insights into their experiences of the project's Fitbit and sleep hygiene advice intervention. They also provided valuable insights into sleep and exercise in the context of their lives. Participants highly valued effective night time sleep and sufficient physical activity; they identified various factors associated with the effects on mood and wellbeing. They described the impact of not having effective night time sleep and sufficient physical activity. The interviews revealed a comprehensive understanding of the links between sleep, exercise, wellbeing, and health. COVID-19 restrictions and impact on exercise in this group were noted, and are important to recognise because some restrictions may have effect on future behaviour and may be in place in the longer term.

Following the implementation of the intervention participants were able to employ lifestyle changes to their daily routine that enabled more effective sleep. The improvement observed in self-management, health and wellbeing are paramount as they may lead to long term and sustained improvements in sleep. This can benefit mental and physical health; improving disease disability-adjusted life years (DALY) and increasing life expectancy [7] [8].

Patients increased their physical activity and exercise due to their use of Fitbit and its software apps. Furthermore, patients reported that Fitbit and its apps provided feedback and objective information, and increased motivation and awareness. Taking action to do physical exercise and changing to a more active lifestyle is especially important because of the links with physical and mental health in a psychosis population; building sufficient physical activity into daily/weekly routine long term can reduce DALY and improve life expectancy [5] [3] [18].

The knowledge gained through the interviews regarding both barriers and facilitators of effective sleep and engagement in physical activity is useful for individuals experiencing psychosis, those supporting them, as well as healthcare services. This information can be valuable when identifying and overcoming barriers as well as applying facilitators for positive change when working with people in the context of EIP services [10]. It is useful for mental healthcare workers to know that most people have a good understanding of the links between sleep, exercise, wellbeing, and health; allowing them to effectively engage with people around these topics.

\section{Limitations}

Study limitations include the relatively small sample size and the potential lack of generalisability. People who agreed to participate were self-selected. A level of bias may exist in terms of those agreeing to participate having a more positive experience of the intervention and to be currently experiencing fewer psychotic symptoms than the EIP population average. Twelve is an appropriate number for a qualitative in-depth interview study as saturation often occurs at around 12 
participants in relatively homogeneous groups [19]. The sample herein was a fairly homogeneous group in terms of diagnosis; although some participants have had additional mental illness diagnosis to psychosis, which is not infrequent in an early psychosis population. The participants all attended a single EIP service reducing generalisability.

\section{Conclusion}

Regular physical activity can prevent many chronic medical conditions and is associated with improved cognition, functioning, as well as mental and physical health in people who experience symptoms of psychosis [3] [5]. Healthy night time sleep duration can reduce risk for mortality, diabetes, cardiovascular disease, stroke, coronary heart disease, and obesity [7] [8]. Therefore, in the context of early psychosis, where people often lack effective sleep and have insufficient physical activity [1] [2] [9] [10], it is important for mental health services to offer interventions which can improve sleep and levels of physical activity. This paper has reported the benefits of a relatively simple and low cost intervention for EIP service patients, specifically the provision of a Fitbit, sleep hygiene advice and one to one engagement with a clinician. This is an intervention which can be introduced relatively easily to all EIP services. Furthermore, it could potentially be beneficial to people experiencing other psychiatric disorders too.

\section{Conflicts of Interest}

No other authors have any conflicts of interests to declare.

\section{References}

[1] Soundy, A., Wampers, M., Probst, M., De Hert, M., Stubbs, B., Vancampfort, D., Ströhle, A., et al. (2013) Physical Activity and Sedentary Behaviour in Outpatients with Schizophrenia: A Systematic Review and Meta-Analysis. International Journal of Therapy and Rehabilitation, 20, 588-595. https://doi.org/10.12968/ijtr.2013.20.12.588

[2] Vancampfort, D., Guelinkcx, H., Probst, M., Stubbs, B., Rosenbaum, S., Ward, P.B. and De Hert, M. (2015) Associations between Metabolic and Aerobic Fitness Parameters in Patients with Schizophrenia. The Journal of Nervous and Mental Disease, 203, 23-27. https://doi.org/10.1097/NMD.0000000000000229

[3] Rhodes, R.E., Janssen, I., Bredin, S.S., Warburton, D.E. and Bauman, A. (2017) Physical Activity: Health Impact, Prevalence, Correlates and Interventions. Psychology \& Health, 32, 942-975. https://doi.org/10.1080/08870446.2017.1325486

[4] Schuch, F., Vancampfort, D., Firth, J., Rosenbaum, S., Ward, P., Reichert, T., Stubbs, B., et al. (2017) Physical Activity and Sedentary Behavior in People with Major Depressive Disorder: A Systematic Review and Meta-Analysis. Journal of Affective Disorders, 210, 139-150. https://doi.org/10.1016/j.jad.2016.10.050

[5] Mittal, V.A., Vargas, T., Osborne, K.J., Dean, D., Gupta, T., Ristanovic, I., Shankman, S.A., et al. (2017) Exercise Treatments for Psychosis: A Review. Current Treatment Options in Psychiatry, 4, 152-166. https://doi.org/10.1007/s40501-017-0112-2

[6] NICE (2014). Psychosis and Schizophrenia in Adults: Prevention and Management. 
https://www.nice.org.uk/guidance/cg178/chapter/1-Recommendations\#care-acrossall-phases

[7] Chaput, J.P., McNeil, J., Després, J.P., Bouchard, C. and Tremblay, A. (2013) Short Sleep Duration as a Risk Factor for the Development of the Metabolic Syndrome in Adults. Preventive Medicine, 57, 872-877. https://doi.org/10.1016/j.ypmed.2013.09.022

[8] Jike, M., Itani, O., Watanabe, N., Buysse, D.J. and Kaneita, Y. (2018) Long Sleep Duration and Health Outcomes: A Systematic Review, Meta-Analysis and Meta-Regression. Sleep Medicine Reviews, 39, 25-36. https://doi.org/10.1016/j.smrv.2017.06.011

[9] Klingaman, E.A., Palmer-Bacon, J., Bennett, M.E. and Rowland, L.M. (2015) Sleep Disorders among People with Schizophrenia: Emerging Research. Current Psychiatry Reports, 17, Article No. 79. https://doi.org/10.1007/s11920-015-0616-7

[10] Davies, G., Haddock, G., Yung, A.R., Mulligan, L.D. and Kyle, S.D. (2017) A Systematic Review of the Nature and Correlates of Sleep Disturbance in Early Psychosis. Sleep Medicine Reviews, 31, 25-38. https://doi.org/10.1016/j.smrv.2016.01.001

[11] American Psychiatric Association (2013) Diagnostic and Statistical Manual of Mental disorders (DSM-5 ${ }^{\circledR}$ ). American Psychiatric Pub. https://doi.org/10.1176/appi.books.9780890425596

[12] Chum, J., Kim, M.S., Zielinski, L., et al. (2018) Acceptability of the Fitbit in Behavioural Activation Therapy for Depression: A Qualitative Study. Evidence-Based Mental Health, 21, 76. https://doi.org/10.1136/ebmental-2017-102763corr1

[13] Beattie, Z., Oyang, Y., Statan, A., Ghoreyshi, A., Pantelopoulos, A., Russell, A. and Heneghan, C. (2017) Estimation of Sleep Stages in a Healthy Adult Population from Optical Plethysmography and Accelerometer Signals. Physiological Measurement, 38, 1968. https://doi.org/10.1088/1361-6579/aa9047

[14] Naslund, J.A., Aschbrenner, K.A., Barre, L.K. and Bartels, S.J. (2015) Feasibility of Popular m-Health Technologies for Activity Tracking among Individuals with Serious Mental Illness. Telemedicine and e-Health, 21, 213-216. https://doi.org/10.1089/tmj.2014.0105

[15] Aledavood, T., Torous, J., Hoyos, A.M.T., Naslund, J.A., Onnela, J.P. and Keshavan, M. (2019) Smartphone-Based Tracking of Sleep in Depression, Anxiety, and Psychotic Disorders. Current Psychiatry Reports, 21, Article No. 49. https://doi.org/10.1007/s11920-019-1043-y

[16] NICE (2016) Implementing the Early Intervention in Psychosis Access and Waiting Time Standard: Guidance.

https://www.nice.org.uk/guidance/qs80/resources/implementing-the-early-interven tion-in-psychosis-access-and-waiting-time-standard-guidance-2487749725

[17] Braun, V. and Clarke, V. (2006) Using Thematic Analysis in Psychology. Qualitative Research in Psychology, 3, 77-101. https://doi.org/10.1191/1478088706qp063oa

[18] Hjorthøj, C., Stürup, A.E., McGrath, J.J. and Nordentoft, M. (2017) Years of Potential Life Lost and Life Expectancy in Schizophrenia: A Systematic Review and Meta-Analysis. The Lancet Psychiatry, 4, 295-301. https://doi.org/10.1016/S2215-0366(17)30078-0

[19] Guest, G., Bunce, A. and Johnson, L. (2006) How Many Interviews Are Enough? An Experiment with Data Saturation and Variability. Field Methods, 18, 59-82. https://doi.org/10.1177/1525822X05279903 